\title{
Formations of Class and Gender | Des femmes respectables. Remarques sur la traduction en sciences sociales
}

Formations of Class and Gender / Des femmes respectables. Notes on

Translation in Social Science

Formations of Class and Gender / Des femmes respectables. Comentarios sobre la traducción en ciencias sociales

\section{Marie-Pierre Pouly}

\section{(2) OpenEdition \\ Journals}

Édition électronique

URL : http://journals.openedition.org/bssg/119

DOI : $10.4000 /$ bssg. 119

ISSN : 2490-9424

Éditeur

Presses universitaires de Vincennes

Référence électronique

Marie-Pierre Pouly, «Formations of Class and Gender | Des femmes respectables. Remarques sur la traduction en sciences sociales », Biens Symboliques / Symbolic Goods [En ligne], 1 | 2017, mis en ligne le 15 octobre 2017, consulté le 04 mars 2021. URL : http://journals.openedition.org/bssg/119 ; DOI : https://doi.org/10.4000/bssg. 119 

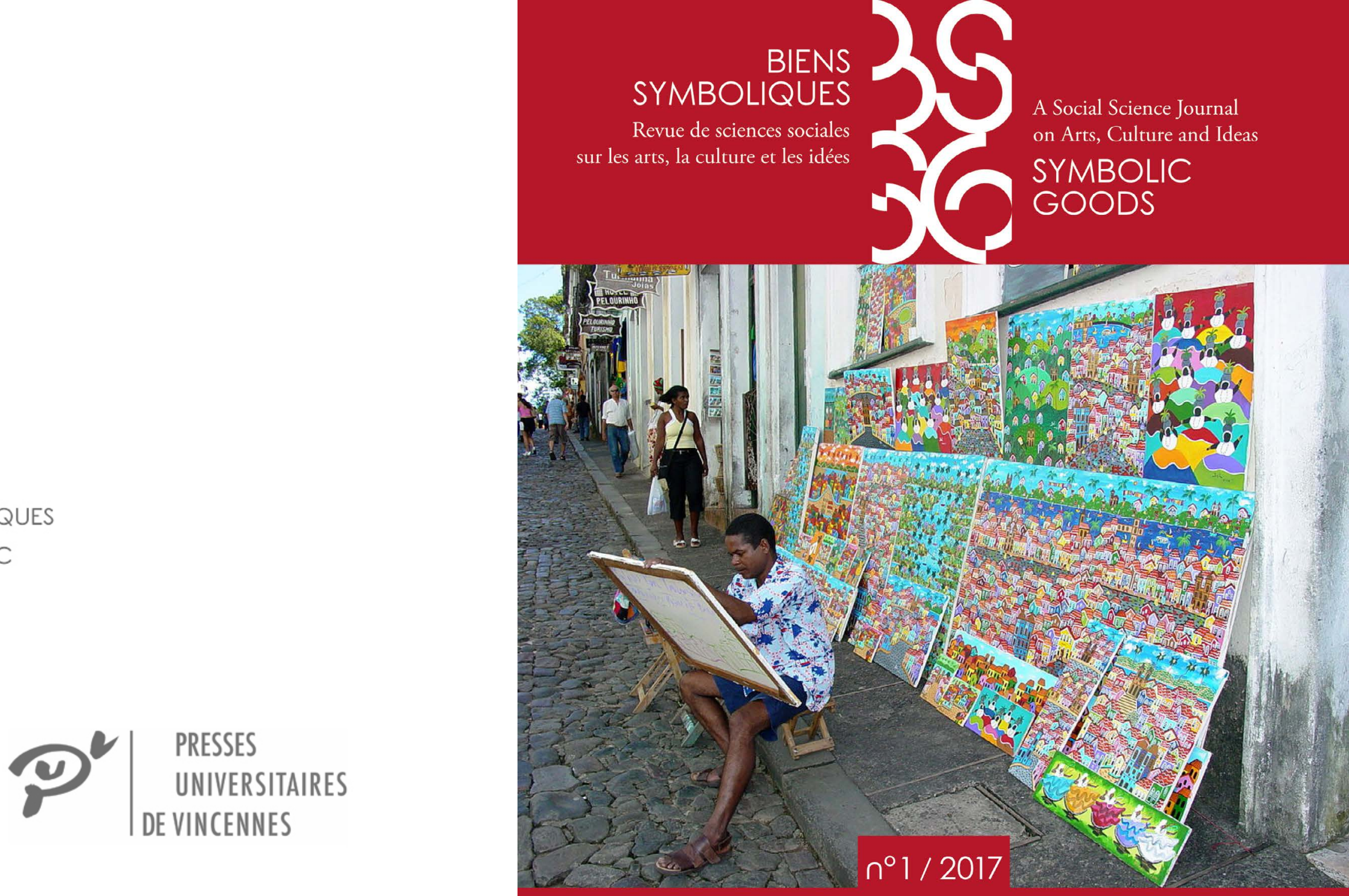

\section{Artistes ordinaires \\ Ordinary Artists}




\title{
Formations of Class and Gender | Des femmes respectables. Remarques sur la traduction en sciences sociales
}

\author{
Marie-Pierre Pouly
}

En revenant sur la traduction de Formations of Class and Gender [Des femmes respectables], de Beverley Skeggs, que j'ai faite pour la maison d'édition Agone (Skeggs 2015 [1997]), je voudrais d'abord contribuer à lever le voile sur certains processus de traduction en sciences sociales qui ne sont généralement pas explicités bien qu'ils soient lourds de conséquences dans la réception des textes traduits. À travers cette expérience, il s'agit de mettre au jour un ensemble d'éléments qui déterminent les choix de traduction, en distinguant ce qui relève du fonctionnement des langues source et cible (langue courante, académique, sociologique), ce qui dépend d'une configuration éditoriale spécifique et ce qui renvoie, enfin, aux propriétés des traducteurs et traductrices - et plus largement des passeurs du texte - et à leur position dans le champ des sciences sociales. J'expliciterai d'abord la configuration éditoriale de la traduction de l'ouvrage de Beverley Skeggs en la comparant à d'autres configurations de traduction ; j'exposerai ensuite les logiques qui ont conduit à opter pour différentes options de traduction, relatives notamment à la langue scientifique, au cadre théorique mobilisé par l'auteure, à la notion de caring ou à la classe sociale'.

1 Je remercie Celia Bense Ferreira Alves et Séverine Sofio pour leurs commentaires qui ont grandement contribué à clarifier mes réflexions, ainsi que Sylvain Laurens qui a vérifié l'exactitude de mes souvenirs tout en encourageant cette démarche de dévoilement des coulisses de la traduction. 
Première de couverture de la traduction de l'ouvrage de Beverley Skeggs (2015). Des Femmes respectables. Classe et genre en milieu populaire. Traduit de l'anglais par Marie-Pierre Pouly

\section{Des femmes respectables}

Classe et genre en milieu populaire

Traduit de l'anglais par Marie-Pierre Pouly

Préface d'Anne-Marie Devreux

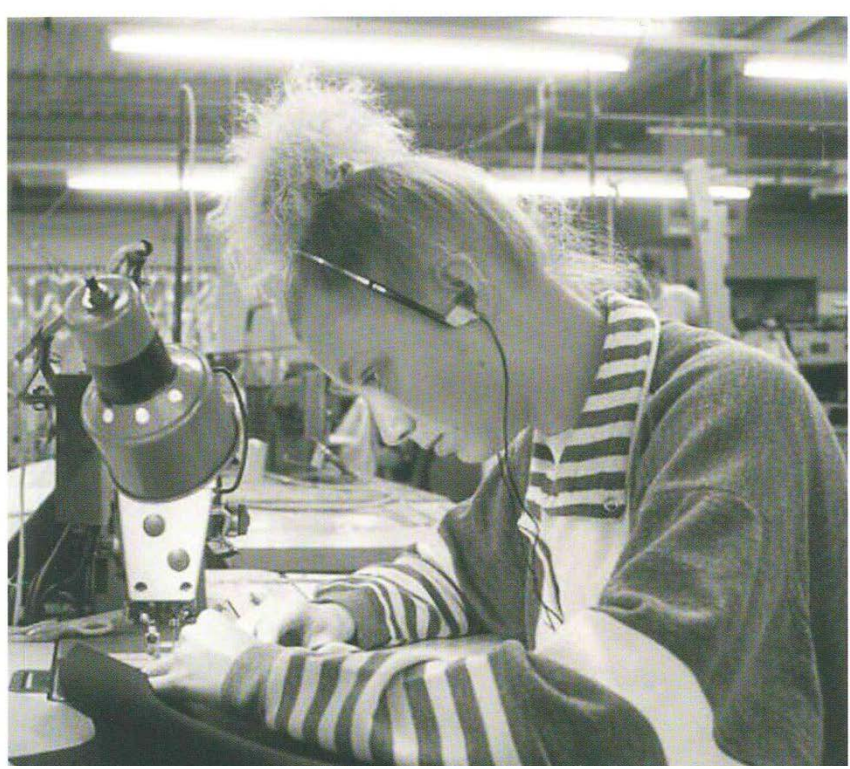

Première de couverture de l'édition originale de l'ouvrage de Beverley Skeggs (1997). Formations of Class and Gender. Becoming respectable

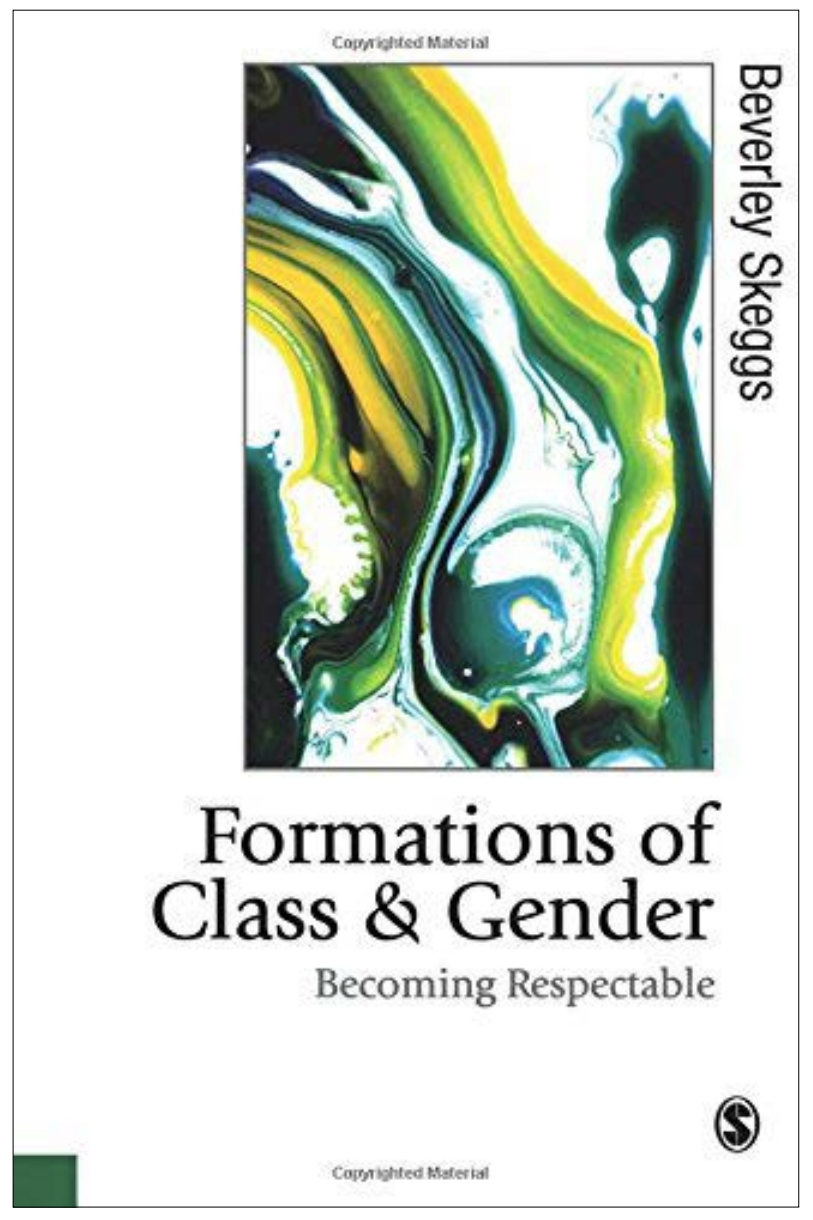

(c) Sage Publishing. 


\section{Configuration éditoriale et position de la traduction}

\subsection{Système éditorial et place des traducteurs}

La traduction des textes de sciences sociales - pour simplifier le propos, on se concentre ici sur le cas des ouvrages - s'inscrit dans différentes configurations éditoriales qui produisent des effets variables sur le texte final et sa réception et sur la visibilité des traducteurs et traductrices et de leurs options traductives. Ces configurations se distinguent d'abord par la place respective et chronologique qu'occupent les linguistes spécialistes de traduction et les sociologues ${ }^{2}$. On peut spécifier les relations entre ces deux types de spécialistes ou de compétences en distinguant, analytiquement, trois types de configurations, chaque type se subdivisant en deux possibilités.

Dans le premier cas de figure, un seul des deux types de spécialistes est représenté, qu'elle ou il soit linguiste ou spécialiste de sciences sociales. À titre d'exemple, on peut citer L'École des ouvriers, l'ouvrage de Paul Willis traduit de l'anglais par Bernard Hoepffner, spécialiste de la traduction de textes littéraires anglophones (Willis 2011 [1978]). Le deuxième cas est aujourd'hui le fait de spécialistes de sciences sociales qui connaissent très bien la langue traduite. On peut ainsi évoquer les traductions d'Erving Goffman, de Joseph Gusfield, ainsi que d'Elihu Katz et Paul Lazarsfeld par le sociologue Daniel Céfaï (Goffman 2013 [1963], Gusfield 2009 [1981], Katz \& Lazarsfeld 2008 [1955]).

2 J'utiliserai dans ce texte le terme de sociologue de façon générique pour faire référence aux spécialistes de sciences sociales au sens large.
Dans le deuxième ensemble, des relectures ou des révisions sont effectuées a posteriori, la traduction des linguistes pouvant être révisée par des spécialistes de sciences sociales, mais des spécialistes de sciences sociales peuvent aussi solliciter une relecture par des linguistes. Dans le premier cas, citons par exemple Family fortunes, de Leonore Davidoff et Catherine Hall, traduit par Christine Wünscher (linguiste traductrice) (Davidoff \& Hall 2014 [1987]). La traduction a été entièrement révisée par la sociologue Isabelle Clair, qui a établi en outre une note sur la traduction, tandis que l'ensemble était relu par Fabrice Bensimon, angliciste spécialiste d'histoire britannique. Dans le second cas, mentionnons par exemple Outsiders, l'ouvrage d'Howard Becker traduit de l'américain par les sociologues Jean-Pierre Briand et Jean-Michel Chapoulie, la traduction étant relue par l'angliciste Marie-Christine Mopinot (Becker 1985 [1963]) ${ }^{3}$.

Dans le troisième ensemble, les compétences sociologiques et linguistiques sont étroitement associées à toutes les étapes du processus de traduction, soit parce que sociologues et linguistes collaborent tout au long de la traduction, soit parce que la traductrice ou le traducteur est à la fois linguiste et spécialiste de sciences sociales. Citons dans le premier cas la traduction de Street Corner Society de William Foote Whyte, à laquelle ont collaboré des anglicistes comme Jean Sévry et des sociologues ou anthropologues, avec une préface d'Henri Peretz (Whyte 1996 [1943]). Dans le deuxième cas, on peut évoquer les traductions distinctes de Max Weber par Isabelle Kalinowski (Weber 2005 [1919]) et Jean-Pierre Grossein (Weber 2006), ou la traduction de Tally's Corner

3 Relecture signalée en note de bas de page. 
par Celia Bense Ferreira Alves (Liebow 2010 [1967]). C'est cette dernière configuration qui caractérise la traduction de l'ouvrage de Beverley Skeggs : angliciste de formation, j'étais à l'époque de la traduction devenue enseignante et chercheure en sociologie.

Dans les faits, la position dans ces configurations n'est pas univoque. Des linguistes traduisant sans relecture de spécialistes de sciences sociales ont acquis une fine connaissance des sciences sociales ; des sociologues sans titre scolaire de linguiste ou formation spécifique de traduction ont acquis des réflexes de traduction au fil de leurs lectures et traductions : elles ou ils connaissent généralement très bien la langue traduite et sont souvent bilingues par socialisation familiale et/ou professionnelle. De plus, il faut souligner le travail souvent invisible de relecture, à l'aune des sciences sociales, opéré par les directeurs et directrices de collection ou éditrices et éditeurs, et celui, souvent non explicité, de préfaciers ou postfacières issus des sciences sociales. Dans le cas de la traduction de Paul Willis aux éditions Agone, qui sera évoquée à plusieurs reprises (Willis 2011 [1978]), bien que la précision n'apparaisse pas dans l'ouvrage, deux directeurs de collection, sociologues, ont longuement retravaillé la version finale de la traduction, en lien avec le traducteur. D'une façon générale, les éditeurs et éditrices soulignent l'important travail de relecture qu'ils doivent souvent consacrer aux traductions (Noël 2012a).

En deuxième lieu, ces configurations varient en fonction de l'appareil éditorial conçu par l'éditeur ou l'éditrice pour orienter la réception de l'ouvrage traduit et la place qu'accorde le péritexte à divers « passeurs ». Dans les configurations éditoriales qui prévoient un appareil critique pour mettre l'ouvrage en contexte et souligner son intérêt sociologique, ce sont généralement les sociologues ayant traduit l'ouvrage qui l'introduisent, le préfacent ou le postfacent ; dans d'autres cas, elles ou ils commentent l'ouvrage traduit par d'autres (après avoir éventuellement révisé la traduction). Les rares cas où les traducteurs ou traductrices linguistes commentent l'ouvrage sont ceux qui correspondent à la dernière des configurations décrites plus haut, où le traducteur est à la fois linguiste et spécialiste de sciences sociales.

Selon les configurations, la place et la visibilité des traducteurs et traductrices et de leurs choix traductifs sont ainsi plus ou moins grandes. On peut penser que c'est quand les traducteurs sont eux-mêmes sociologues que se pose de façon la plus visible la question de l'effet de leur propre position sur les choix de traduction. Elles ou ils occupent, dans le champ des sciences sociales vers lequel le texte est traduit, une position qui, malgré des homologies possibles, est inévitablement différente de celle que l'auteur.e du texte traduit occupe ou occupait dans le champ dans lequel elle ou il s'inscrivait : la traduction comporte nécessairement une part de transposition vers un certain état du champ des sciences sociales.

La question des choix traductifs est en réalité toujours opérante quelle que soit la configuration, mais elle n'accède pas de façon identique à la visibilité selon les cas. Faute de connaître les débats sédimentés au sein des sciences sociales des deux langues et champs nationaux en jeu dans la traduction, les traducteurs linguistes risquent toujours de prendre position dans le champ des sciences sociales en toute ignorance de cause, si l'on peut dire, et éventuellement de façon contradictoire. Du côté des traducteurs issus des sciences 
sociales, il convient non pas de distinguer celles et ceux qui traduiraient depuis une position spécifique tandis que d'autres seraient « neutres », dans une traduction transparente, mais plutôt de distinguer les degrés d'explicitation de l'incidence de leurs choix de traduction sur le raisonnement sociologique (et réciproquement).

Ces choix sont notamment liés, d'une part, à la position de la maison d'édition et à ses ambitions éditoriales et, d'autre part, à celle du ou des passeurs. Leur entreprise traductive poursuit des intentions dans le champ des sciences sociales, en faisant exister certaines positions scientifiques (certains concepts, certaines conceptions de la recherche, de l'ethnographie, certaines façons d'écrire, etc.), tout en ayant des effets - plus ou moins consciemment escomptés - sur la position de la maison d'édition comme sur la carrière des passeurs, traducteurs et traductrices inclus, associés aux entreprises de traduction.

\subsection{Configuration éditoriale pour Des femmes respectables}

La position de la maison d'édition et de la collection, les différents passeurs impliqués dans cette traduction et leurs relations, ainsi que certaines caractéristiques de la traductrice situent la configuration éditoriale dans laquelle a été publié l'ouvrage Des femmes respectables.

Les éditions Agone, fondées en 1998, sont une maison d'édition indépendante. Leur ligne éditoriale se veut soucieuse des luttes contemporaines et soumise aux exigences du savoir. Le projet éditorial correspond avant tout à un projet politique en proposant des œuvres censées fournir au plus grand nombre des outils d'émancipation à travers la compréhension du monde social et de ses formes de domination. L'ouvrage de Beverley Skeggs y est publié dans la collection « L'ordre des choses », créée en 2011 et dirigée par Sylvain Laurens, Julian Mischi et Étienne Pénissat. On peut noter que Learning to Labour, l'ouvrage de Paul Willis publié dans cette collection en 2011, comme Formations of Class and Gender, avait antérieurement fait l'objet d'un article dans Actes de la recherche en sciences sociales (Cartier 2012). La revue, fondée par Pierre Bourdieu, donne une indication de la position dans le champ des sciences sociales depuis laquelle Agone entendait traduire ces ouvrages tout en signalant une forme de pré-consécration permettant de parier sur ces ouvrages sans trop de risques.

L'investissement bénévole d'universitaires au sein de ces maisons d'édition indépendantes « critiques », sous la forme notamment de direction de collections, permet, comme les traductions, d'accumuler du capital symbolique (Noël 2012a). Cesuniversitairestententalors de tenir une double lignecritique et scientifique, en s'inscrivant dans le champ des sciences sociales tout en cherchant à rendre accessibles des textes engagés, au sens où ils mettent en lumière des mécanismes de domination. En publiant des livres d'universitaires mais aussi des livres de militants ou de journalistes, Agone occupe une position intermédiaire (Noël 2012a). " Mieux comprendre les logiques qui régissent le monde social est un préalable nécessaire pour s'inscrire contre "l'ordre des choses" ${ }^{\prime \prime}$ : c'est l'idée que défend la collection éponyme dans laquelle l'ouvrage de Skeggs a été publié.

4 Source : présentation de la collection sur le site de la maison d'édition. 
En 2012, 38 \% des ouvrages du catalogue d'Agone étaient des traductions (hors titres littéraires), alors que dans le secteur des sciences humaines, la part des traductions dans la production globale était passée de $6 \%$ en 1986 à $4,5 \%$ en 2000 (Noël 2012b : 276). Comme le note Sophie Noël, Agone peut se positionner, à l'instar d'autres éditeurs critiques indépendants, sur des niches peu exploitées ou délaissées par les éditeurs plus établis, telles que la traduction d'ouvrages d'intellectuels prestigieux dans le domaine des sciences humaines et sociales, avec une prise de risque limitée car les aides publiques (ville, conseil général, régional, DRAC, $\mathrm{SNE}, \mathrm{CNL}$, etc.) ou privées pèsent d'un poids important dans l'économie de ces petites structures caractérisées par de faibles ressources financières (Noël 2012a).

L'ouvrage de Beverley Skeggs, publié en 1997 chez Sage sous le titre Formations of Class and Gender. Becoming Respectable, rend compte d'une enquête ethnographique de douze ans réalisée, dans le cadre d'une thèse, entre les années 1980 et le début des années 1990. Agone avait obtenu en 2013 un financement pour traduire un ouvrage sur le genre issu des sciences sociales britanniques et choisi de traduire l'ouvrage de Beverley Skeggs, en partie pour faire pendant à l'ouvrage de Paul Willis, L'École des ouvriers (Willis 2011/1978), traduit et publié peu avant dans la même collection («L'ordre des choses »).

Sachant que je faisais lire un chapitre de l'ouvrage dans le cadre d'un enseignement de sociologie, Sylvain Laurens, maître de conférences en sociologie à l'EHESS et codirecteur de la collection «L'ordre des choses », m'a alors proposé de traduire l'ouvrage. II voyait en moi (m'a-t-il confié a posteriori) trois caractéristiques qui lui semblaient pouvoir éviter les déconvenues auxquelles la collection avait été confrontée : en premier lieu, la double compétence de sociologue et de traductrice, en second lieu, une compatibilité avec le projet éditorial d'Agone, et en troisième lieu une sensibilité à l'engagement féministe. Angliciste de formation, donc exercée à la traduction universitaire, comme à l'explicitation des options traductives, familière depuis mes études d'anglais des analyses en termes de genre, j'ai enseigné dans un Institut d'études politiques où je partageais avec plusieurs collègues un intérêt pour certains travaux des Cultural studies de langue anglaise. Je me suis engagée dans une reconversion à la sociologie en mobilisant un certain nombre de schèmes d'analyse des travaux développés par et autour de Pierre Bourdieu, tout en appréciant les travaux d'ethnographie menés, par exemple, autour du département de sciences sociales de l'École normale supérieure, et les travaux défendant une conception unitaire des sciences sociales, publiés dans des revues comme Genèses ou Actes de la recherche en sciences sociales.

Après l'habituel essai de cinq pages demandé aux traducteurs, Agone m'a confié la traduction et Sylvain Laurens en a assuré le suivi. Nous avons été collègues dans deux établissements successifs et entretenons des relations de sympathie intellectuelle et amicale, ce qui détermine en partie la collaboration éditoriale étroite qui s'est nouée tout au long de la traduction, et l'accord parfois à demi-mots qui s'établissait entre nous sur les enjeux globaux de la traduction de l'ouvrage comme sur les choix spécifiques opérés au fil du texte. 
Dans le cadre de nos discussions régulières sur l'ouvrage, Sylvain Laurens m'a proposé de rédiger une postface. Au fil de la traduction et à son issue, j'ai rédigé un commentaire sur l'ouvrage qui mêlait notes de traduction et mise en perspective analytique de l'ouvrage. Jugeant le texte trop long et trop lourd pour une postface dans une collection qui entend rendre les textes qu'elle publie accessibles à un public non universitaire, Sylvain Laurens m'a proposé de le scinder, en réorganisant les éléments qui forment la postface finalement publiée, tout en conservant pour une publication ultérieure ce qui relevait de la note de traduction. Sylvain Laurens a, en outre, confié à Anne-Marie Devreux, sociologue spécialiste du genre, le soin d'introduire l'ouvrage par une préface mettant en perspective les travaux de Beverley Skeggs et l'ouvrage traduit, tandis qu'Anne-Marie Devreux et Sylvain Laurens conduisaient un entretien avec Beverley Skeggs, placé après la traduction et avant la postface.

Cette traduction, comme le péritexte qui l'accompagne, est ainsi le fruit d'un dialogue engageant l'auteure de l'ouvrage dans certains cas, moi-même comme traductrice, la maison d'édition, des co-directeurs de collection et la relectrice d'Agone qui est également traductrice de l'allemand (Marie Hermann). Une collègue sociologue agrégée de lettres, Valérie Duguet, a également relu l'ouvrage traduit. Le titre relève d'un long dialogue entre la maison d'édition, la relectrice d'Agone, la préfacière Anne-Marie Devreux et moimême. À l'issue de ce travail de relecture, le texte traduit, ainsi que l'entretien, ont été envoyés à Beverley Skeggs, qui les a validés.

\section{Adaptation des langues, choix éditoriaux et position des passeurs}

Les choix de traduction opérés au fil du texte-dans l'isolement du travail quotidien de la traductrice ou dans un dialogue continu avec la maison d'édition, les relectrices et parfois l'auteure - relèvent analytiquement de trois cas de figure. Ils dépendent, d'abord, de l'adaptation d'un système langagier à un autre. Ils découlent, ensuite, d'un contexte éditorial spécifique, différent d'une traduction à une autre. Ils sont sous-tendus, enfin, par la position des passeurs (traducteur ou traductrice, ou universitaires investis dans l'entreprise de traduction).

\subsection{L'adaptation des systèmes langagiers}

Premièrement, les choix de traduction correspondent à la nécessaire adaptation d'une langue à une autre. Ils découlent ainsi à la fois d'un sens pratique de traduction, des conventions de traduction sédimentées dans la formation des traducteurs ou dans des manuels d'enseignement de la traduction ${ }^{5}$ et, enfin, du travail de contextualisation effectué en amont (recherches sur l'auteure traduite, son œuvre, ses pratiques rédactionnelles).

5 Pour citer les ouvrages sans doute les plus mobilisés dans l'enseignement de la traduction auprès des anglicistes français, voir notamment, par ordre chronologique, Vinay \& Darbelnet (1958), Guillemin-Flescher (1981), Chuquet \& Paillard (1987) et Ballard (1994) 
L'ouvrage de Beverley Skeggs donne essentiellement à entendre deux langues différentes ${ }^{6}$ : la langue des femmes enquêtées dont sont restitués des extraits d'entretien, d'une part ; la langue académique des sciences sociales, d'autre part. Ces deux registres linguistiques fonctionnent différemment en anglais et en français, et l'adaptation, quand elle a des incidences sur le raisonnement sociologique, exige des connaissances en sciences sociales.

La temporalité de l'écriture et de la traduction implique également des changements de registres. En effet, la thèse puis l'ouvrage sont rédigés dans les années 1990 et restituent une enquête un peu antérieure, tandis que le livre est traduit en 2015. Cela pose donc la question de la temporalité de textes correspondant à des états distincts des champs respectifs des sciences sociales, d'une part, et des sociolectes des femmes étudiées, d'autre part. À quel état du langage des sciences sociales et des sociolectes convient-il d'être fidèle ? Faut-il, par exemple, trouver un équivalent du langage familier des années 1980, au risque de « ringardiser » ou de folkloriser les propos des femmes, ou bien des homologies avec un sociolecte plus contemporain ${ }^{7}$ ?

Les langues savantes sont, on le sait, loin d'être uniformes. Les traducteurs et les traductrices de sciences sociales tentent de transmettre avec la plus grande précision possible

6 On peut y ajouter une langue plus littéraire, ressortissant du registre autobiographique, dans certains brefs passages où Beverley Skeggs relate, dans l'introduction et la conclusion, son expérience d'enquête en mobilisant des éléments biographiques tout en faisant état de ses émotions et de ses espoirs.

$7 \quad$ Les spécialistes de la traduction de textes classiques de périodes éloignées ont coutume de dire qu'il faut les retraduire tous les trente ans. un raisonnement intellectuel solidaire d'une manière de l'exprimer, d'un style consubstantiel. C'est donc à une réflexion sur la manière d'écrire que se livrent celles et ceux qui sont chargés d'exprimer la pensée dans son style. La langue savante conceptuelle de Harrison White (White 2011 [1992]), celle, élégante, logique et analytique de Goffman (Goffman 2013 [1963]), les « torsions » butlériennes du langage scientifique ordinaire (Butler 2004 [1997]), la langue épique de Du Bois (Du Bois 2004 [1920]), celle de Levine, entrelardée d'érudition et d'anecdotes parlantes (Levine 2010 [1988]), celles, accessibles, de Peter Berger (Berger 2006 [1963]) ou d'Howard Becker (Becker 2002 [1998]), qui ne souhaitent pas se prendre trop au sérieux, n'entraînent pas le même travail traductif, ni les mêmes transpositions. Le respect des intentions auctoriales n'implique pas pour autant une traduction littérale : la fidélité au style conduit souvent, au contraire, à se déplacer d'un pas, à transposer, pour produire des effets homologues, comme en témoignent les débats sur la traduction des virgules de Proust (Sapiro 2012 : 48). La langue de Beverley Skeggs dans Formations of Class and Gender est essentiellement une langue ethnographique et analytique, à la fois précise et claire, sans fioritures, parfois ironique et combative. Elle se trouvait donc ajustée aux intentions éditoriales.

\subsection{Les intentions éditoriales : à propos de la lisibilité}

Les choix de traduction dépendent, en deuxième lieu, du contexte éditorial dans lequel l'ouvrage est traduit, qui entraîne une transposition plus ou moins importante. Dans le cas de l'ouvrage de Beverley Skeggs, on l'a vu, une partie 
des choix éditoriaux vise à faciliter la lisibilité du texte, en travaillant la langue de l'enquête de terrain pour privilégier les effets d'intelligibilité produits par le texte, et donc les effets espérés d'émancipation, plutôt qu'une démarche plus érudite de mise à disposition pour les universitaires francophones d'un témoignage sur la façon dont s'écrivaient les sciences sociales britanniques dans les années 1980-1990.

D'une façon générale, la traduction privilégie donc la lisibilité et la fluidité de la démonstration sociologique. Aussi, tout en respectant l'exactitude de la traduction et de ses nuances, certaines phrases ont été coupées (jeu sur la ponctuation), tandis qu'en d'assez rares occasions, l'ordre des propositions au sein des paragraphes ou l'ordre des paragraphes ont été modifiés, dans l'intérêt de la démonstration. Dans un autre ordre d'idées, alors que le prétérit domine la restitution ethnographique dans le texte en langue anglaise, c'est le présent, et parfois le passé composé ou l'imparfait, qui sont privilégiés en français, plutôt que le passé simple, plus « noble », souvent choisi par les traducteurs littéraires quand ils traduisent des récits, qu'ils soient ou non ethnographiques. Le présent a pour effet d'entraîner les lecteurs dans l'immédiateté de l'enquête et de son analyse tout en évitant les effets de littérarisation de l'expérience associés au passé simple.

Pour des raisons identiques de lisibilité, et après en avoir discuté avec Beverley Skeggs, une modification substantielle a été apportée à la structure de l'ouvrage : le chapitre 2 de l'ouvrage anglais a été réduit et déplacé. Des passages écrits dans un style plus abstrait, portant sur le statut de l'expérience et les points de vue situés, qui interrompaient l'élan introductif et retardaient l'accès à la restitution de l'enquête, ont été traduits puis finalement supprimés de l'ouvrage français. Sylvain Laurens et moi-même avons fait cette proposition à Beverley Skeggs, en mettant en avant les intentions de la collection et de la maison d'édition (mettre à disposition des textes de sciences sociales de sorte qu'ils puissent être lus par des non universitaires), mais aussi la cohérence globale de la démarche proposée dans l'ouvrage. En effet, le dernier chapitre sur le féminisme s'interroge sur les droits d'entrée symboliques que risquent d'ériger les textes féministes, en rendant inaccessibles, à celles et ceux qui ne disposent pas du capital culturel nécessaire, les armes qu'ils voudraient pourtant fournir au plus grand nombre. Ce raisonnement, développé par l'auteure, nous semblait très ajusté à l'esprit éditorial de la collection : la réorganisation du chapitre 2 anglais nous semblait ainsi congruente avec le propos de l'ouvrage. Une partie de ce chapitre 2 (sections : «Processus de recherche et élaboration théorique » et «Connexions : différences et similitudes "), concernant notamment le rapport biographique de Beverley Skeggs à son objet de recherche et aux enquêtées, a été déplacée dans l'introduction, ce qui correspond aux pratiques introductives usuelles de restitution des enquêtes de terrain actuellement en vigueur. Enfin, la partie du chapitre 2 initial qui proposait un retour réflexif sur les interprétations contenues dans l'ouvrage a été intégrée à la conclusion. 


\subsection{Connaissances spécialisées et position dans le champ des sciences sociales}

En troisième lieu, les options de traduction engagent des connaissances sociologiques et, dans certains cas, la position personnelle du traducteur ou de la traductrice dans le champ de la sociologie. Qu'il s'agisse du choix de traduire caring et care systématiquement, comme on l'expliquera ci-après, de ne pas tirer la traduction vers un système conceptuel uniquement lié aux études de genre, de retrouver la langue de Pierre Bourdieu en français dans les passages où Beverley Skeggs n'a pas l'intention de la modifier, de transposer la langue académique anglaise vers son équivalent français, à chaque fois, un ensemble de raisonnements sociologiques sont mis en œuvre et susceptibles de varier selon les caractéristiques des traducteurs. La partie qui suit illustre ces raisonnements, tout en donnant à voir combien les différents choix effectués relèvent de façon souvent simultanée des trois éléments que je viens d'évoquer : adaptation des langues, choix éditoriaux et position des passeurs.

\section{Options de traduction}

Sans prétendre épuiser l'ensemble des choix de traduction opérés au fil du texte, cette partie s'organise, pour des raisons de clarté, autour de deux thèmes centraux de l'ouvrage traduit : le travail de soin et les dispositions au dévouement, d'abord, la définition objective et subjective de l'appartenance de classe, ensuite. Elle se clôt, enfin, par quelques remarques sur le métalangage sociologique utilisé.

\subsection{Traduire caring/care avec soin}

Les traductions se heurtent souvent, on le sait, à la question de l'intraduisibilité de certains mots, concepts, expressions, pour plusieurs raisons. Celle-ci découle notamment de la spécificité historique d'une configuration sociale dont certains phénomènes ne trouvent pas d'équivalent dans la société vers laquelle on traduit. Elle provient également du sémantisme de certains termes traduits par d'autres lexies qui, dans la langue d'arrivée, ne recouvrent pas l'ensemble des traits sémantiques d'origine. Elle résulte, enfin, des usages conceptuels spécifiques que font les sociologues de certains termes.

Tout au long de l'ouvrage, Beverley Skeggs utilise les termes de caring (majoritairement) et de care (moins souvent) ainsi que leurs dérivés, dans de nombreux contextes, s'agissant notamment des dispositions, socialement construites comme féminines, à se soucier des autres, s'agissant aussi du travail fréquemment non rémunéré de soin et d'aide dans un contexte domestique ou familial au sens large, mais encore des enseignements de type familialiste (enseignement ménager puis économie domestique) fin $\mathrm{XIX}^{\mathrm{e}}$-début $\mathrm{XX}{ }^{\mathrm{e}}$ siècle puis d'aide à la personne à la fin $d u x^{e}$ siècle. On peut se figurer sur un axe l'étendue sémantique de caring/care, opposant les dispositions morales dévouées (construites comme féminines) les plus profondément incorporées à l'identité subjective (caring self) à un autre pôle correspondant au travail technique d'aide et de soin (caring work). Cette double face du terme contribue à son ambivalence et au fait qu'il est difficile de mettre à distance les dispositions éthiques dans le travail concret d'aide. Entre les deux, les cours d'aide 
et de soin de l'enseignement ménager puis sanitaire et social (caring courses) transmettent des manières de faire techniques, dessinent un horizon professionnel ou familial, et valorisent, renforcent ou inculquent un soi dévoué (caring self).

II n'existe pas de terme unique en français qui couvrirait l'ensemble du sémantisme de caring/care. Au lieu de conserver en anglais et en italiques dans le texte français le terme de care et ses dérivés (care, caring, care for, care about, care worker, caring selves, caring performances, etc.), j'ai pris le parti de toujours traduire. Qu'il me soit permis d'évoquer ici mes réticences à l'égard du choix de ne pas traduire, sans jeter le discrédit sur les formes d'intelligibilité permises par le terme de care et les travaux ethnographiques qu'il a suscités ${ }^{8}$. Nombreux sont les ouvrages ou articles sur « le care » qui commencent par rappeler l'intraduisibilité du concept, leurs auteur.e.s se chargeant d'éclairer le public francophone sur sa signification. Deux choses me gênaient dans cette approche. J'y décelais, dans certains cas, une stratégie distinctive par l'importation d'un terme désigné comme incommensurable, intraduisible, nécessitant l'exégèse de passeurs ${ }^{9}$. L'exégèse

8 Cette notion permet de « décloisonner et de penser ensemble toute une série d'activités qui ont en commun d'être réalisées soit par des femmes, soit par des populations migrantes, minoritaires, et d'être considérées comme des professions subalternes et non qualifiées, dont on ne pourrait pourtan pas se passer. [] [D]ans ces activités, il y a non seulement des tâches matérielles, pénibles, rebutantes, mais aussi tout un travail d'attention aux besoins des autres [] » (Delphy \& Molinier 2012 : 306).

9 Sur la circulation mondiale de catégories savantes le plus souvent retranscrites (non traduites) et les stratégies d'import-export conceptuel, voir Bourdieu \& Wacquant (1998). Pour une défense de l'intérêt de la circulation internationale de certains concepts comme genre/gender ou care, lire Delphy \& Molinier (2012) prouve pourtant que le terme est traduisible, à condition d'accepter que ses divers signifiés puissent être traduits par différents termes selon les contextes. La plupart des auteurs, y compris celles et ceux qui défendent la nature intraduisible de care, optent finalement pour une solution à mi-chemin, où le mot care est utilisé quand il renvoie très explicitement au concept associé à un ensemble de débats théoriques, et traduit quand il trouve aisément, en contexte, un synonyme en anglais. II peut aussi être traduit pour éviter des lourdeurs syntaxiques ou des répétitions. Une deuxième dimension de ma réticence initiale agissait au moment de la traduction de façon plus inconsciente. Elle relève de la formation que j'ai reçue, comme linguiste angliciste, et peut-être plus généralement au sein d'un système d'enseignement français qui inculque le goût des belles lettres ${ }^{10}$, où conserver un terme non traduit ou le franciser revient à une erreur scolaire (l'anglicisme) ou une faute (de goût).

Les phénomènes désignés par Beverley Skeggs en ayant recours aux mots caring et care ne sont en rien intraduisibles ou irréductibles au contexte britannique : ils trouvent le plus souvent leur pendant, avec des dénominations diverses, dans la société française (parmi d'autres). On peut penser d'ailleurs que Skeggs sur-emploie les mots care et caring. D'une part, elle les utilise pour désigner, de façon anachronique, des formations du XIX $\mathrm{X}^{\mathrm{e}}$ siècle dont les dénominations ont pourtant également changé au fil du temps en Grande-Bretagne, comme en France. D'autre part, elle les emploie pour unifier les différentes facettes des relations de service auxquelles les jeunes femmes sont destinées par leur position et leur

10 Voir à ce sujet Casanova (2016) 
formation ; rien n'empêche de faire de même avec des mots français suffisamment récurrents pour produire cet effet d'unification : j'alterne dans la traduction aide/aidante, soin et dévouement/dévouée dont la répétition produit à la longue, me semble-t-il, le même effet de rassemblement.

Le concept de care, tel qu'il a été « reçu » en France - celui d'Arlie Hochschild, Joan Tronto, Evelyn Nakano Glenn ou dans une moindre mesure Carol Gilligan - est d'abord un concept américain, autour duquel s'était déjà formée une école théorique quand Beverley Skeggs écrivait son livre. Cependant, d'une part, Skeggs ne se réfère pas à ces auteures sur le care et, d'autre part, le terme qu'elle utilise le plus fréquemment est caring, soit comme nom abstrait, soit comme adjectif épithète ou attribut, suivi en fréquence décroissante par care, care for, care about comme verbes (plutôt que comme nom abstrait). Le mot care et ses dérivés étant usuels, les emplois du mot par Beverley Skeggs ne sont pas tous, loin de là, conceptuellement chargés au sens où elle utiliserait un concept issu d'une tradition théorique reconnaissable. Utiliser le nom care, et sans doute également son dérivé caring, conduirait ainsi à une affiliation à un corpus théorique que ne revendique pas l'auteure au moment de la rédaction de son ouvrage.

Du fait qu'une bonne partie des occurrences du radical carprennent une forme adjectivale et verbale, le maintien du terme anglais aurait posé un problème insoluble de fluidité linguistique, l'emprunt non francisé étant envisageable pour les noms communs mais quasiment impossible dans la langue académique pour les adjectifs ou les verbes (dire " le care/caring » est acceptable, mais pas " elles carent », " elles sont caring »). Or, sauf dans les cas où caring et care étaient des noms communs abstraits à dimension conceptuelle potentielle, rien ne justifiait a priori d'accorder plus d'importance aux occurrences nominales, qui étaient d'ailleurs plus rares que les autres, en les faisant accéder au stade conceptuel sans traduction alors que les autres occurrences seraient systématiquement traduites.

En anglais, care est entendu comme un terme polysémique, de même qu'en français, les signifiés de « soin » ou d'« aide » sont, selon les contextes, relativement divers (et pourraient être traduits par des termes différents en anglais) : « prendre soin de soi », « soin aux personnes », «travailler avec soin », « soins cosmétiques », « aide aux devoirs », « aide de camp », « aide-soignante », « aide financière », « aide informatique ». Par conséquent, conserver en français les termes care ou caring revient à écraser leur polysémie (dont les importateurs et importatrices du concept soulignent paradoxalement la richesse) puisqu'un locuteur francophone n'entend plus le contenu sémantique qu'un locuteur anglophone entend dans son emploi en contexte. En d'autres termes, une traduction qui ne traduirait pas ou quasiment pas caring/care produirait dans la langue d'arrivée un effet différent de la version originale puisqu'en bloquant le sémantisme de care, on n'entend plus dans le mot que la référence au débat théorique universitaire. Il faut rappeler en outre que ce ne sont jamais tous les signifiés d'un terme polysémique, mais seulement certains d'entre eux, qui sont activés dans un contexte donné, ce qui permet d'ailleurs de traduire en donnant à entendre ce qu'un locuteur de la langue d'origine comprend à chaque occurrence.

Cependant, en multipliant les expressions qui traduisent les termes originaux de care et caring, les lecteurs et lectrices ne sont plus en mesure d'éprouver son ubiquité dans le texte 
original, ni les réseaux de signification que le même terme prend suivant les contextes. Une première solution consistait à faire suivre d'un astérisque chacune des 421 traductions des lexies du radical car- (caring, careful, cared for, etc.) : par exemple, « dévouement* ». Cela avait l'intérêt de transmettre en français le sémantisme activé dans chaque occurrence de caring/care tout en faisant apparaître, pour les lectrices et les lecteurs francophones, l'ubiquité de ce radical dans le texte anglais ; mais cela rendait illisibles de nombreux passages saturés d'astérisques, en contradiction avec les exigences éditoriales d'accessibilité. II était par ailleurs envisageable d'expliciter ces choix et le champ sémantique de car- dans une note sur la traduction plus longue, distincte du corps du texte, ou intégrée à la postface que j'ai rédigée pour l'ouvrage (Pouly 2015). Mais cette solution alourdissait à son tour l'appareil éditorial. La solution finalement retenue a consisté à exposer dans une note de traduction brève (en bas de page) l'étendue polysémique de caring/care dans le texte anglais, dès sa première occurrence et de signaler entre crochets la toute première traduction du terme [caring] dans le corps du texte. On voit à nouveau à travers cet exemple que les solutions de traduction retenues composent inévitablement avec des logiques éditoriales.

La décision de ne pas traduire care est parfois justifiée par l'idée qu'elle permettrait de ne pas choisir entre des traditions analytiques déjà existantes, comme le « courant canguilhemien » pour le champ lexical du « soin », ou les «éthiques ricœuriennes " pour la « sollicitude » (Delphy \& Molinier 2012). Ce choix conduit à se rattacher exclusivement à un corpus théorique existant : celui, justement, du care, et parfois de ses usages dans le champ politique. Du fait de la variété des traductions proposées en activant les différents sens pris en contexte par caring/care dans l'ouvrage de Beverley Skeggs, il me semble que la traduction échappe à l'affiliation à un registre spécifique, quoique l'ethnographie de Beverley Skeggs, en anglais comme en français, puisse aisément être lue à la lumière des schèmes d'analyse du care. Je me suis déplacée par rapport à l'opposition entre soin et sollicitude, en utilisant d'un côté plutôt les lexiques du soin et de l'aide, pour souligner le travail d'aide et les gestes techniques qui ne relèvent que partiellement du domaine médical, et de l'autre côté, en évitant celui de sollicitude, aux connotations religieuses, et en employant plutôt les termes de dévouement et de souci des autres, que Skeggs forge en renversant la notion de «souci de soi » de Michel Foucault.

\subsection{Traduire la classe}

L'ouvrage de Beverley Skeggs documente de façon à la fois historique et ethnographique la position objective de classe et le rapport subjectif à la position sociale des femmes étudiées. D'un point de vue historique, Skeggs s'appuie essentiellement sur les travaux des historiennes et des historiens britanniques de la bourgeoisie qui ont souligné la dimension sexuée de la formation des classes sociales (Davidoff \& Hall 2014 [1987]). Dans son versant ethnographique, Des femmes respectables donne à voir la coexistence de la fidélité à soi et de l'assimilation à l'idéal dominant, dans la double conscience que vivent les femmes étudiées. Elles éprouvent le jugement classant du goût des classes supérieures jusque dans leurs pratiques les plus intimes et leurs choix esthétiques. Leur goût est à double ressort : leur goût de classe est aussitôt doublé d'un (pres) 
sentiment de son indignité culturelle. II s'agit ainsi d'insister plutôt sur la conscience double et l'ambivalence omniprésente dans la façon dont les pratiques sont éprouvées.

\subsubsection{Working class et middle class}

La société anglaise et la société française ne sont pas structurées de la même manière et les catégorisations sociales, avec des signifiants linguistiques changeants, ont varié dans le temps. Les découpages ne se superposent pas : à la différence de " classes moyennes ", " middle class » est aujourd'hui un terme qui englobe des catégories supérieures en France (professions libérales, professions intellectuelles notamment), tandis qu' « upper class » désigne les personnes qui possèdent un titre de noblesse et n'est pas souvent employée pour parler des classes supérieures dans leur ensemble. En outre, les catégories utilisées à diverses périodes pour désigner un groupe du point de vue de l'analyse sociologique (notamment) ne correspondent pas forcément aux termes utilisés par les membres du groupe pour s'auto-désigner (classes populaires vs classe ouvrière). Enfin, ces décalages peuvent être différents dans les deux sociétés : working class est en anglais un terme courant d'auto-appellation, une catégorie de la nomenclature, de l'appareil statistique comme de l'analyse sociologique.

Dans le texte anglais, Beverley Skeggs joue sur une opposition binaire entre working class et middle class, qui reconduit l'opposition classique entre « nous » et « eux ». Pour la période contemporaine, et alors qu'elle emprunte à La Distinction (Bourdieu 1979), Beverley Skeggs n'a pas recours à une opposition ternaire (classes populaires, petite bourgeoisie/ classe moyenne, bourgeoisie/classes supérieures), alors que les femmes « middle class » désignées par les enquêtées sont souvent bourgeoises, comme en témoigne l'adjectif qui revient dans les entretiens pour décrire leur style "bourge » (posh). Dans le cas français, l'expression « classe moyenne » au singulier est synonyme de bourgeoisie dans la première moitié $\mathrm{du} \mathrm{XIX}^{\mathrm{e}}$ siècle mais prend un sens tout autre en se déclinant au pluriel (classes moyennes) à la fin du XIX $X^{\mathrm{e}}$ siècle, pour désigner l'ensemble situé en dessous de la bourgeoisie et au-dessus du peuple (Baudelot, Establet, Malmort 1974 ; Charle 1991). Dans le cas anglais, pour le $\mathrm{XIX}^{\mathrm{e}}$ siècle, il faut entendre par middle class la bourgeoisie par opposition à la gentry et l'aristocratie. De ce fait, nous avons traduit middle class par bourgeoisie pour le $\mathrm{XIX}^{\mathrm{e}}$ siècle ${ }^{11}$ et le plus souvent par classes supérieures (ou classes moyennes et supérieures, selon le contexte) pour la période de l'enquête de Beverley Skeggs, en utilisant l'adjectif bourgeois pour les deux périodes. II faut donc entendre dans le texte français, par « classes supérieures », le « eux » de Hoggart, ceux qui ne sont pas des classes populaires.

Le nom working class a été traduit le plus souvent par «classes populaires » au pluriel, selon l'usage le plus répandu de la sociologie française contemporaine, sauf dans quelques cas, pour le $\mathrm{XIX}^{\mathrm{e}}$ siècle, et dans certains des passages où les femmes discutent avec Beverley Skeggs de leur sentiment d'appartenance de classe. Une partie des réponses montrent que le mot working est parfois interprété littéralement, pour désigner le travail, auquel cas « classe ouvrière » est plus pertinent.

11 Pour des choix similaires voir Davidoff \& Hall (2014 [1987] : 17-21). 
"La vraie classe ouvrière, c'est ceux que tu vois faire la queue pour toucher les allocs. Ils sont crades et pauvres et ils ont pas de boulot, enfin je suppose que peut-être ils bossent si c'est la classe ouvrière [working class], hein, peutêtre qu'ils bossent. Si c'est la classe ouvrière, ils doivent être ouvriers et donc travailler hein je suppose, dans des boulots pourris » (Sheenah 1992).

L'adjectif working class a été traduit par l'adjectif « populaire ». En français, le terme «populaire » peut s'appliquer aux groupes sociaux qui occupent les positions les plus basses dans la distribution économique et symbolique (les groupes/ classes populaires) ; il peut aussi désigner des pratiques culturelles envisagées dans leur écart ou leur opposition à des pratiques perçues comme savantes, légitimes ou distinguées. En anglais, on peut utiliser «lowbrow » pour désigner les pratiques les moins savantes, mais Beverley Skeggs n'utilise jamais cet adjectif ni, plus généralement l'opposition lowbrowl highbrow, qui désigne des distinctions au sein des pratiques culturelles fondées sur le langage (radio, télévision, écrit, etc.) et dont l'historien Lawrence Levine étudie la genèse (Levine 2010 [1988]). Le texte de Beverley Skeggs fait plutôt entendre l'opposition « bourge » ou " classe »/vulgaire (posh/classy vs vulgar) qui s'applique aux corps ou aux objets (habillement, ameublement).

\subsubsection{Sociolecte}

Les nombreux extraits d'entretien, qui constituent l'une des richesses du livre, par leur caractère révélateur et l'usage qu'en fait Beverley Skeggs, ont été traduits en faisant entendre le langage familier, tout en laissant percer, notamment pour les entretiens des femmes « installées » à la fin de l'enquête, les formes de correction du langage qui sont précisément l'une des façons d'être « respectable » pour soi et pour autrui, et d'effacer les stigmates de classe. Si l'on garde à l'esprit les croisades de la middle class anglaise contre l'intempérance linguistique, on peut penser que la correction du langage s'impose aux femmes qu'étudie Beverley Skeggs autant que la surveillance de l'apparence et des dispositions au soin, avec un schème d'opposition fort/doux se traduisant par l'opposition entre le langage fort masculin et le langage féminin modéré (en accord avec la « gentillesse » et le calme requis par le travail d'aide et de soin). Les travaux de sociolinguistes suggèrent que cette correction s'impose au moins sur les marchés linguistiques les plus surveillés. Beverley Skeggs, figure familière mais également universitaire et ancienne enseignante de certaines enquêtées, suscitait sans doute un type de langage intermédiaire en la matière.

Il fallait donc tenir un style oral et dans le même temps relativement contrôlé syntaxiquement et lexicalement : par exemple, il n'y a aucun " fuck » ou « fucking », seulement quelques «bloody» (traduit par «putain de », qui est plus fort que bloody mais l'est moins que fucking). Les mots plus grossiers apparaissent pour exprimer le ressentiment à l'égard des formes de domination.

" Je me suis dit, mais qu'est-ce qu'on fout là, genre, tu sais, venir au lycée pour baigner des poupées, quoi, ça te résume bien le truc là, hein ? Tu vas pas au lycée pour baigner des poupées, c'est ça que je veux dire, jouer avec des putains de poupées [bloody dolls], quoi. J'ai 17 ans, et j'aimais même pas les poupées quand j'avais 7 ans » [Cindy, Services médicaux, $O$ levels]. 
Beverley Skeggs est très sensible à l'idée que ses représentations savantes puissent apporter de l'eau au moulin des représentations conservatrices qui renvoient les femmes des classes populaires à leur absence de respectabilité. J'ai évité, comme le fait Beverley Skeggs, toute transcription semi-phonétique qui, si elle peut donner à entendre l'accent de classe, évoque aussi les conventions littéraires et condescendantes de représentation du peuple en introduisant, par le décalage avec l'usage écrit ordinaire, une forme d'exotisme. Néanmoins, je me suis également gardée d'une transcription trop écrite qui aurait suggéré une forme d'hyper-correction. J'ai ainsi effacé certaines syllabes non prononcées (« vous pouvez pas me rabaisser », «y avait », " toute façon »). Certains entretiens sont faits avec des jeunes femmes de seize ans encore scolarisées : il fallait faire sentir les manières collectives de parler, faire entendre les expressions d'une langue commune du groupe des pairs qui paraisse crédible (j'ai pensé à la langue de jeunes femmes françaises occupant une position homologue et dont je connaissais les manières de parler).

Je n'ai pas, cependant, cherché à respecter strictement la véracité historique du parler populaire des jeunes femmes blanches des années 1980 occupant une position similaire en France. Certaines tournures sont donc légèrement plus récentes et connotent un langage « jeune » - « c'était trop facile », «genre », « style », « elle vit chez les bisounours »mais aucune n'est plus ancienne. D'un point de vue éditorial, il s'agissait de mettre à disposition une enquête ethnographique vivante en privilégiant, plutôt que le témoignage historique, la force percutante des paroles des enquêtées, sans stigmatiser les femmes par l'effet qu'aurait pu produire une langue située historiquement, pouvant paraître datée.

\subsection{Métalangage sociologique}

J'ai employé de façon préférentielle le métalangage sociologique commun en vigueur dans les sciences sociales françaises au moment de la traduction ${ }^{12}$. Ainsi, je n'ai recouru au métalangage spécifique d'une tradition théorique (celui de Pierre Bourdieu, de Michel Foucault, de Judith Butler, etc. - « capital », « souci de soi », « performance ») que quand l'auteur était explicitement ou implicitement présent dans l'analyse. De même, quand plusieurs traductions étaient possibles, j'ai fait le choix d'expressions et de formules qui font partie de la langue sociologique commune de l'époque. Certains choix reflètent néanmoins la langue sociologique de la traductrice (" schèmes d'interprétation ", " sociogenèse ", « les méthodes sont des théories en acte », etc.).

\subsubsection{Spécificités de la langue académique anglaise}

Quoique certains choix de traduction, liés aux spécificités de la langue source et de la langue cible, soient rarement commentés, ils engagent le raisonnement sociologique. Ainsi, la facilité avec laquelle la langue anglaise permet des tournures passives est particulièrement adaptée à un type de raisonnement sociologique qui souligne le poids des structures sans désigner spécifiquement les agents qui exercent une contrainte. En français, tous les manuels de traduction conseillent de préférer les formes actives pour éviter l'apparente lourdeur des formes passives, en introduisant par

12 C'est également le choix que défendent Frédéric Godart et Michel Grossett concernant leur traduction de l'ouvrage d'Harrison C. White, Identité et contrôle (Godart \& Grossetti 2011). 
exemple un « on » impersonnel ; mais le « on » pose problème dans les assertions sociologiques (il suggère l'existence d'agents tout en gardant le flou sur leur identité). J'ai opté le plus souvent pour des tournures impersonnelles (subjectivity is generated > la subjectivité s'élabore, s'engendre, etc.) et conservé certains passifs.

La langue anglaise est également plus souple dans la composition lexicale, permettant de fabriquer de nombreuses lexies par adjonction d'affixes sur les racines saxonnes, qui sonnent « simples » (à l'inverse du vocabulaire académique issu du fonds gréco-latin, avec ses syllabes plus nombreuses et qui est, pour des raisons sociales puissantes et anciennes liées à l'usage du latin comme du français, perçu à la fois comme plus profond intellectuellement, plus prestigieux et plus aristocratique). La souplesse de la composition lexicale anglaise permet de créer des termes à mi-chemin entre l'expression indigène et la notion sociologique, dans des formulations très ramassées qu'il faut expliciter dans la traduction et donc étoffer, comme la classlessness ${ }^{13}$, que l'on peut traduire par le fait de ne pas s'identifier à une classe sociale, de ne pas éprouver un sentiment d'appartenance de classe, autrement dit de ne pas se percevoir à travers des catégories de classe ; même chose pour la workingclassness, le fait de s'identifier à la classe ouvrière/aux classes populaires.

La langue anglaise permet enfin plus facilement qu'en français des formulations neutres du point de vue du genre (Beverley Skeggs n'utilise que des formulations neutres), car

13 Agrégation de deux suffixes : -less : suffixe négatif (sans) ; -ness : nom abstrait l'adjectif n'est pas fléchi en fonction du genre, tandis que les cas de flexion des professions (comme le suffixe féminin -ess) sont plus rares qu'en français. C'est surtout à propos des noms composés en man - type fireman - et des pronoms de troisième personne du singulier reprenant des noms neutres (a theorist - neutre ou indifférencié - traditionnellement repris par he, pronom masculin) que la question s'est posée aux féministes de langue anglaise. II a été proposé des noms composés en woman ou indifférenciés (firewoman ou firefighter) et une reprise inclusive par s/he (possible à l'écrit), they (singulier), he or she ou she, ou un évitement en amont du pronom de troisième personne du singulier, en remplaçant par exemple le groupe nominal antécédent au singulier par un pluriel générique (Chevalier, de Charnay, Gardelle 2017 : 15). Ces luttes ont conduit à une application assez généralisée des recommandations inclusives, dans la plupart des textes publiés, mais aussi assez couramment à l'oral où la reprise par he or she est fréquente. Aux facteurs linguistiques limitant l'androcentrisme de la langue anglaise et facilitant la réforme des graphies s'ajoute le fait que ce caractère androcentré limité est perçu et combattu de longue date (Chevalier, de Charnay, Gardelle 2017 : 13). A contrario, la langue française impose encore aujourd'hui une flexion des noms, des pronoms et des adjectifs en fonction du genre et ne permet la neutralisation qu'en attirant, le plus souvent, l'attention sur la neutralisation opérée. Par exemple, l'expression feminist theorist permet de désigner une personne (de genre non déterminé) qui produit des théories féministes. En français, l'expression anglaise peut se traduire notamment par « théoricien féministe » (masculin supposé neutre, selon l'usage voulu par l'Académie française), «théoricien·ne féministe » (neutralisation rendue 
visible), «théoricien(ne) féministe » (neutralisation rendue visible mais avec mise entre parenthèse du féminin) ou encore « théoricienne féministe » (dé-neutralisation avec introduction de l'idée que les théoriciennes féministes sont forcément des femmes ; ou, dans certaines interprétations, coup de force linguistique consistant à imposer que le féminin, humain prototypique, l'emporte dans tous les cas d'indétermination, mais avec le risque que les personnes ignorant cet usage l'interprètent comme une forme de détermination renvoyant à une théoricienne en particulier).

Chacune de ces options correspond à une position spécifique. II fallait donc imaginer quelle position Beverley Skeggs choisirait si elle se trouvait elle-même prise dans cet ensemble de choix (étant entendu que si elle était sociologue de langue française, elle combattrait le caractère androcentré de la syntaxe de langue française $)^{14}$. J'ai globalement opté pour une rédaction infidèle aux prescriptions de l'Académie française, en rejetant en outre la solution qui consiste à mettre entre parenthèses le féminin. J'ai suivi les guides de rédaction épicène ${ }^{15}$ qui me paraissaient les plus satisfaisants d'un point de vue stylistique : trouver une expression inclusive en français quand cela est possible (« les universitaires féministes ») ; donner le féminin en premier et mettre le masculin entre parenthèses quand la probabilité est plus grande que la personne désignée par le nom soit une femme (« une théoricienne (un théoricien) féministe » ; « avec

14 Pour une présentation des diverses stratégies existantes, lire Daniel Elmiger (Elmiger 2017).

15 Le langage épicène évite l'utilisation par défaut du genre masculin qui marginalise le genre féminin dans l'ordre linguistique et contribue donc, dans l'ordre symbolique, aux processus de domination masculine. celles (ceux) qui... ») ; donner, par ordre alphabétique, quand cela n'ajoute pas trop de lourdeur syntaxique (donc pas systématiquement : une ou deux fois par paragraphe) à la fois le féminin et le masculin («les théoriciennes et les théoriciens », " les traducteurs et les traductrices », " elles et ils », " celles et ceux », etc.). Il s'agissait d'éviter, en tout cas, la situation paradoxale de traductions se voulant « radicales » du point de vue de la pensée du genre, comme celles de Judith Butler, et qui n'hésitent pourtant pas à reconduire un langage androcentré en français ${ }^{16}$. Sous la plume du traducteur Maxime Cervulle, « theorist » est ainsi traduit par le féminin « théoricienne » quand il s'agit de théoriciennes ou de théoriciens du genre tandis qu'à la même page, les mêmes theorists mais d'obédience lacanienne ont droit au masculin («théoriciens lacaniens ») (Butler $2006: 60$ ).

\subsubsection{Performance ou distance au rôle}

Le vocabulaire de la mise en scène et de la manifestation de manières d'être (faire féminine, manifester sa respectabilité ou mettre en scène son dévouement) est très présent dans l'ouvrage de Beverley Skeggs. Dans le texte anglais, ce sont les mots performance/to perform et display qui sont le plus souvent utilisés, souvent en alternance : ils laissent entendre qu'il y a du jeu entre les positions occupées et les dispositions, que des rôles sont endossés et que l'ajustement aux positions n'est ni immédiat, ni évident, ni entier. Le terme de performance

16 "Comment traduire Judith Butler ? Comment rejouer sa "provocation" (excitable speech) en français ? " se demandent Jérôme Vidal et Charlotte Nordmann, qui ont traduit son ouvrage, dans une note introductive sur la traduction (Butler 2004 : 7). 
est utilisé par Judith Butler, citée par Beverley Skeggs, en lien avec une théorie de la performativité des identités, et généralement traduit en français par « performance ». Le verbe perform est traduit par accomplir ou par mettre en scène dans la traduction de Charlotte Nordmann, qui fait cependant suivre régulièrement la traduction par le mot perform entre parenthèses (Butler 2004 [1997]).

Cependant Beverley Skeggs ne fait qu'une fois explicitement référence à l'usage qu'en fait Judith Butler (au sens de réitération performative des normes) et son usage du terme de performance se démarque la plupart du temps de celui de Butler, de même qu'elle dialogue de façon critique avec la notion de mascarade (notion travaillée par Judith Butler). J'ai donc choisi de le rendre le plus souvent par « mise en scène »/ " mettre en scène », et « manifester ». Le terme français fait entendre les formes de distance au rôle et de dédoublement suggérées dans le texte de Skeggs : les femmes étudiées sont souvent amenées à adopter des manières de faire qui ne correspondent ni à leur socialisation ni à leurs conditions d'existence, par exemple des normes de soin et d'aide ou des types de féminité qui leur sont initialement étrangères et qu'elles endossent, le plus souvent sous surveillance. Elles peuvent être critiques dans les coulisses, par exemple dans les discussions entre elles et avec Beverley Skeggs, ne pas se reconnaître, tout en adoptant ces manières de faire et en y adhérant plus ou moins fortement : ainsi, les dispositions exigées pour le soin sont plus difficiles à mettre à distance car elles déterminent la valeur sur le marché du travail comme sur le marché matrimonial.

\subsubsection{Faut-il multiplier les capitaux ?}

L'usage de l'expression « cultural capital » dans le texte anglais ne correspond pas souvent (26 fois sur 65) à l'usage stabilisé du concept en français : elle désigne le plus souvent des traits culturels populaires dont Beverley Skeggs note précisément qu'ils ne font pas capital. Par décision éditoriale et avec l'accord de Beverley Skeggs, j'ai traduit dans ces caslà cultural capital par l'expression plus floue de " ressource culturelle » au singulier ou au pluriel, qui désigne alors un trait culturel qui a de la valeur sur certains marchés, notamment locaux, sans pour autant faire capital sur des marchés ou dans des champs supra-locaux (notamment l'école, le marché du travail ou celui des représentations médiatiques) ${ }^{17}$. Ce point est également abordé dans l'entretien avec l'auteure.

II existe néanmoins dans l'ouvrage deux usages de cultural capital pour lesquels on peut parler (d'une sous-espèce) de capital culturel, auquel cas on a donc gardé « capital culturel » en français. D'abord, dans certains cas, les dispositions féminines au souci des autres, à l'aide et au soin, partiellement institutionnalisées dans des formes scolaires, peuvent fonctionner comme une espèce (relativement faible) du capital culturel, incorporé dans les gestes professionnels de l'aide ou les manières d'élever les enfants, institutionnalisé dans les établissements de soin et d'aide et d'enseignement ou les manuels décrivant les «bonnes pratiques », certifié par

17 On peut rappeler ici la mise au point d'Érik Neveu sur les propriétés de tout capital, assortie d'une proposition de moratoire sur l'invention de nouveaux capitaux : il doit avoir une efficacité (variable selon le temps et l'espace), une convertibilité (inégale) en d'autres types de capitaux, et nécessiter des formes d'appropriation et d'incorporation sous la forme de dispositions incorporées (Neveu 2013) 
des titres scolaires ou des stages, même si ces ressources ne font que faiblement capital et n'offrent, par exemple, que l'accès au marché du travail dit secondaire, précaire et mal rémunéré. D'autre part, Beverley Skeggs mentionne souvent les effets du capital culturel des classes supérieures, des universitaires, des féministes, celui dont les femmes étudiées ne sont pas dotées.

On se trouve dans la plupart des autres cas confronté à un usage décalé du concept de capital culturel, comme dans d'assez nombreux travaux utilisant l'expression en langue anglaise. D'abord, l'adjectif « culturel » est entendu dans son sens culturaliste anthropologique et celui que visent les Cultural studies en se donnant pour tâche de porter au jour, ou de réhabiliter, la richesse et la complexité des cultures populaires. Ensuite, le nom « capital » est entendu dans un sens faible, comme «trait » culturel qui a de la valeur pour le groupe dont il émane, même si ce groupe est dominé et que ses traits culturels sont dévalués sur d'autres marchés et dans d'autres champs. Or si un trait culturel n'est pas légitime, on voit mal comment on pourrait le qualifier de capital culturel : par définition, le capital est du capital s'il fait capital, s'il est efficace. Par ailleurs, dans le texte de Beverley Skeggs et dans d'autres travaux anglophones, on lit parfois que le capital culturel, s'il est légitimé, devient du capital symbolique. Or ce sont les traits culturels qui, s'ils sont légitimés, forment le capital culturel (qui peut, de fait, opérer comme capital symbolique, comme tout autre capital à condition qu'il soit reconnu, qu'il exerce un charisme).

La définition des trois états du capital culturel - incorporé, certifié, institutionnalisé - (Bourdieu 1979b), qui s'est diffusée en langue anglaise par résumés interposés, donne lieu à une autre série de décalages. Au lieu de penser les trois états du capital culturel comme trois formes sous lesquelles le (même) capital peut exister, les trois états sont distingués pour considérer que tout trait culturel incorporé, et tout ce qui relève du corps socialisé (comme les dispositions de genre), les dispositions durables de l'organisme, sont du « capital culturel ». Cette assertion procède d'un renversement (illogique) de la proposition de Pierre Bourdieu : le capital culturel peut exister sous forme de disposition incorporée ; on en conclut que les dispositions incorporées sont du capital culturel (or toutes les dispositions ne correspondent pas à du capital culturel).

\section{Conclusion}

Les significations des œuvres changent même lorsque leur texte ne change pas. Mais il est des cas où c'est dans l'œuvre elle-même, modifiée dans sa littéralité, que l'histoire introduit des variantes textuelles et transformations du sens. Dans une situation comme dans l'autre, ce que la main de l'auteur a écrit n'est que la matrice de variations dont les agents sont multiples (Chartier $2015: 201$ ).

Inséré dans une note de lecture de Des femmes respectables parue dans un magazine « société » en ligne, le commentaire qui suit, suscité par la postface que j'ai écrite pour l'ouvrage et, indirectement, par ma traduction, a le mérite de faire apparaître plusieurs impensés sur la traduction en sciences sociales.

[...] [L]a postface, dont l'auteur, qui est aussi la traductrice de l'ouvrage, utilise du papier pour donner des leçons de 
sociologie à Beverley Skeggs, qui n'a pas compris ceci, qui a fait un contresens sur cela, qui sûrement voulait dire ceci (mais l'a mal dit) ou ne voulait pas dire cela (mais l'a bien dit). On se demande comment l'éditeur a pu laisser passer ce manque de courtoisie, ridicule, qui plus est, cette grossièreté, qui au passage jette un doute sur la fidélité de la traduction, pourtant bien agréable, bien lisible pour le lecteur français [...].

Ce jugement révèle, d'abord, la prégnance du mythe de la traduction transparente. II souligne, ensuite, la position subalterne ou ancillaire conventionnellement attendue du traducteur ou de la traductrice à l'égard de l'auteur.e à chaque étape du processus de traduction, jusqu'à la publication, et l'invisibilité (Venuti 2008) et la neutralité corrélatives qui en sont exigées ${ }^{18}$. Il évoque, enfin, le rôle de l'éditeur.rice dans le renforcement ou le renversement de cette position. À travers cet article, j'ai tenté d'éclairer chacun de ces points en invitant les lecteur.rice.s de textes traduits à se doter d'outils de compréhension des enjeux des choix de traduction.

Alors qu'en littérature, du moins pour les classiques, il est fréquent de disposer de plusieurs traductions d'un même texte, et donc admis que les traducteur.rice-s opèrent des choix qui relèvent de l'interprétation, la grande rareté des traductions multiples d'un même texte en sciences sociales,

18 On peut penser, par ailleurs, que le commentateur estime que les « bonnes manières » universitaires devraient interdire d'engager une discussion scientifique au sein d'une postface. Cette défense des bonnes manières me semble porteuse d'une vision conservatrice des échanges scientifiques, interdisant la discussion spécifique au nom de la politesse, tout en publiant ces préceptes moraux en dehors des espaces de débats réglés où s'imposent d'ordinaire des règles d'argumentation scientifiques. ainsi, sans doute, que la moindre familiarité d'une partie des spécialistes de sciences sociales avec la pratique de traduction, conduit plus aisément à penser que la traduction consiste en une opération relativement simple devant conduire, pour autant que les traducteur.rice-s soient des linguistes suffisamment compétent·e.s, à un texte « fidèle » identique d'un·e traducteur.rice à l'autre. Si la commune maîtrise des conventions traductives et des différences stylistiques des langues en jeu impose de nombreuses solutions de traduction relativement similaires d'une traduction à l'autre ${ }^{19}$, d'autres dimensions relèvent de choix éditoriaux et traductifs qui engagent une connaissance autant qu'une vision des sciences sociales.

Marie-Pierre Pouly

Université de Limoges/GRESCO http://gresco.labo.univ-poitiers.fr/

\section{Bibliographie}

BALLARD Michel (1994). La Traduction de l'anglais au français. $2^{\mathrm{e}}$ édition. Paris, Nathan.

Baudelot Christian, Establet Roger, Jacques Malemort (1974). La Petite-bourgeoisie en France. Paris, Maspero.

BECKER Howard (2002) [1998]. Les Ficelles du métier : comment conduire sa recherche en sciences sociales. Traduit de l'américain par Jacques Mailhos et révisé par Henri Peretz. Paris, La Découverte.

BECKER Howard (1985) [1963]. Outsiders. Traduit de l'américain par JeanPierre Briand et Jean-Michel Chapoulie. Paris, Métaillé.

19 II ne s'agit en aucun cas, on l'aura compris, de défendre l'idée d'une interprétation donnant libre cours à la subjectivité ou à la créativité des traducteurs. Sur ce point, lire Casanova (2016) ; sur la sédimentation des interprétations successives des textes transmis, traduits, revisités, voir Chartier (2015) 
BERGER Peter (2006) [1963]. Invitation à la sociologie. Traduit de l'américain par Christine Merllié-Young, introduction, notes et édition de Dominique Merllié. Paris, La Découverte.

Bourdieu Pierre (1979a). La Distinction. Paris, Minuit.

BOURDIEU Pierre (1979b). "Les trois états du capital culturel ». Actes de la recherche en sciences sociales, $30:$ 3-6.

BOURDIEU Pierre \& WACQUANT Loïc (1998). « Sur les ruses de la raison impérialiste ». Actes de la recherche en sciences sociales, $121: 109-118$. BUTLER Judith (2004) [1997]. Le Pouvoir des mots. Politique du performatif. Traduit de l'américain par Charlotte Nordmann. Paris, Éditions Amsterdam. BUTLER Judith (2006) [2004]. Défaire le genre. Traduit de l'américain par Maxime Cervulle. Paris, Éditions Amsterdam.

CARTIER Marie (2012). "Le caring, un capital culturel populaire ? ». Actes de la recherche en sciences sociales, 191-192 : 106-113.

Casanova Pascale (2016). La Langue mondiale. Paris, Seuil.

CHARLE Christophe (1991). Histoire sociale de la France au XIXe siècle. Paris, Seuil.

CHARTIER Roger (2015). La Main de l'auteur et l'esprit de l'imprimeur. Paris, Gallimard.

Chevalier Yannick, Charnay Hughes (DE), Gardelle Laure (2017). "Bases linguistiques de l'émancipation: système anglais, système français ». Mots. Les langages du politique, $113: 9-36$.

ChUQuet Hélène \& PAILLARD Michel (1987). Approche linguistique des problèmes de traduction anglais-français. Paris, OPHRYS.

DAVIDOFF Leonore \& HALL Catherine (2014) [1987]. Family Fortunes. Hommes et femmes de la bourgeoisie anglaise. 1780-1850. Traduit de l'anglais par Christine Wünscher. Paris, La Dispute.

DELPHY Christine, MolinIER Pascale et alii (2012). « Genre à la française ». Sociologie, 3(3) : 299-316.

Du BoIs William (2004) [1920]. Les Âmes du peuple noir. Traduit de l'américain et commenté par Magali Bessone. Paris, Presses de l'École normale supérieure.

ELMIGER Daniel (2017). «Binarité du genre grammatical - binarité des écritures ? ». Mots. Les langages du politique, $113:$ 37-52.

FOWLER Bridget (2003). «Reading Pierre Bourdieu's Masculine domination. Notes towards an Intersectional Analysis of Gender, Culture and Class ». Cultural studies, 17 (3/4) : 468-494.
GodART Frédéric \& GROSSETTI Michel (2011) [1992]. « La longue histoire d'une traduction impossible ». In WHITE Harrison C., Identité et contrôle. Une théorie de l'émergence des formations sociales : 25-28.

GoffMAN Erving (2013) [1963]. Comment se conduire dans les lieux publics : notes sur l'organisation sociale des rassemblements. Traduit de l'américain et postfacé par Daniel Cefaï. Paris, Economica.

GUILLEMIN-FLESCHER Jacqueline (1981). Syntaxe comparée du français et de l'anglais. Problèmes de traduction. Paris, Éditions OPHRYS.

GUSFIELD Joseph (2009) [1981]. La Culture des problèmes publics. L'alcoo au volant : la production d'un ordre symbolique. Traduit de l'anglais et postfacé par Daniel Cefaï. Paris, Economica.

HoggaRT Richart (1991) [1988]. 33 Newport Street : autobiographie d'un intellectuel issu des classes populaires anglaises. Traduit de l'anglais par Christiane Grignon et Claude Grignon avec la collaboration de Christopher Todd et une présentation de Claude Grignon. Paris, Gallimard/Le Seuil. KATZ Elihu \& LAZARSFELD Paul F. (2008) [1955]. Influence personnelle : ce que les gens font des médias. Traduit de l'américain et postfacé par Daniel Cefaï. Paris, Armand Colin.

LEVINE Lawrence (2010) [1988]. Culture d'en haut, culture d'en bas. L'émergence des hiérarchies culturelles aux États-Unis. Traduit de l'américain par Olivier Vanhée et Marianne Woollven. Paris, La Découverte. Liebow Elliott (2010) [1967]. Tally's corner : les Noirs du coin de la rue. Traduit de l'américain et préfacé par Célia Bense Ferreira-Alves. Rennes, Presses Universitaires de Rennes.

Neveu Érik (2013). «Les sciences sociales doivent-elles accumuler les capitaux ? À propos de Catherine Hakim, Erotic Capital, et de quelques marcottages intempestifs de la notion de capital ». Revue française de science politique, $63: 337-358$.

NoËL Sophie (2012a). L'Édition indépendante critique : engagements politiques et intellectuels. Lyon, Presses de l'Enssib.

NoËL Sophie (2012b). « L'engagement par la traduction. Le rôle des petits éditeurs indépendants dans l'importation des ouvrages de sciences humaines ». In SAPIRO Gisèle (dir.). Traduire la littérature et les sciences humaines. Conditions et obstacles. Paris, Ministère de la Culture et de la Communication : 273-295.

Pouly Marie-Pierre (2015). « Domination culturelle et respectabilité ». In SKEGGS Beverley, Des femmes respectables. Classe et genre en milieu 
populaire [Formations of Class and Gender. Becoming respectable, Sage Publishing]. Traduit de l'anglais par Marie-Pierre Pouly. Marseille, Agone : 359-386.

SAPIRO Gisèle (2012). "Introduction. Les obstacles économiques et culturels à la traduction ». In SAPIRO Gisèle (dir.). Traduire la littérature et les sciences humaines. Conditions et obstacles. Paris, Ministère de la Culture et de la Communication : 25-53.

SkEGGS Beverley (2015) [1997]. Des femmes respectables. Classe et genre en milieu populaire [Formations of Class and Gender. Becoming respectable, Sage Publishing]. Traduit de l'anglais par Marie-Pierre Pouly, Marseille, Agone.

VENUTI Lawrence (2008) [1995]. The Translator's Invisibility. A History of Translation. Oxon, Routlege.

VINAY Jean-Pierre \& DARBELNET Jean (1958). Stylistique comparée du français et de l'anglais. Montréal, Beauchemin.

Weber Max (2005) [1919]. La Science, profession \& vocation. Traduit de l'allemand par Isabelle Kalinowski et suivi de Leçons wébériennes sur la science \& la propagande par Isabelle Kalinowski. Marseille, Agone.

WEBER Max (2006). Sociologie des religions, textes réunis. Textes traduits et présentés par Jean-Pierre Grossein avec une introduction de JeanClaude Passeron ( $2^{\mathrm{e}}$ édition, revue et corrigée). Paris, Gallimard.

WHITE Harrison C. (2011) [1992]. Identité et contrôle. Une théorie de l'émergence des formations sociales. Traduit de l'américain par Frédéric Godart et Michel Grossetti. Paris, EHESS : 25-28.

WHYTE William Foote (1996) [1943]. Street Corner Society : la structure sociale d'un quartier italo-américain. Traduction de l'américain par Suzy Guth, Jean Sevry, Monique Destrade et Jean Destrade et préfacée par Henri Peretz.

WiLLIS Paul (1978). "L'école des ouvriers 》. Actes de la recherche en sciences sociales, $24: 50-61$.

WiLLIS Paul (2011) [1978]. L'École des ouvriers. Comment les enfants d'ouvriers obtiennent des boulots d'ouvriers. Traduit de l'anglais par Bernard Hoepffner. Marseille, Agone. 